\title{
Frequency-Selective Galerkin Models for Turbulent Boundary Layer Drag Reduction *
}

\author{
M.Hakenberg* D.Abel* \\ * RWTH Aachen University, 52056 Aachen, Germany. (e-mail: \\ M.Hakenberg@irt.rwth-aachen.de).
}

\begin{abstract}
Galerkin models for 3D-turbulent boundary layers usually are too complex to be successfully incorporated into model based control strategies. Limiting the frequency spectrum of the ensemble data offers a possibility to reduce the model size and still map the controlrelevant dynamics into the reduced-order model. An adaptation of the standard POD-Galerkin procedure is proposed using the example of a turbulent boundary layer, which is actuated by a periodic volume force. A closed-loop simulation shows an exemplary application of such a reduced order model in a model based control setup.
\end{abstract}

Keywords: Flow control, model reduction, model based control

\section{INTRODUCTION}

Turbulent boundary layer drag reduction offers a potential for energy saving in aeroplanes, trains and other applications with large flow attached surfaces. Turbulent fluctuations close to the wall cause a significant increase in friction drag compared to a laminar flow. These fluctuations are not stochastically independent but follow periodic patterns in time and space, the so-called coherent structures (Holmes et al. [1996]). The disruption of these near-wall cycles has been investigated by passive means such as riblet structures, cf. Walsh [1982], for a long time. However, passive means are limited to a narrow area of operation and may even deteriorate turbulent friction drag outside the design point. Active flow control can be adapted to the current operating conditions, but at the cost of external actuation energy. Therefore, the important factor to be considered is the net-energy balance of the actuated system (Frohnapfel et al. [2012]). Understanding of the near-wall flow physics has to be incorporated into the actuation scheme to achieve a efficient use of actuation energy. The numeric simulations of Choi et al. [1994] and the experimental works from Rathnasingham and Breuer [2003] and Yoshino et al. [2008] are investigations towards a targeted actuation. This actuation aims at the destruction of individual coherent structures. Good results were achieved with that approach, however, for low Reynoldsnumber flows. Things become more difficult for higher Reynolds-numbers, which are relevant for technical applications. Here the size of the turbulent structures reduces to sub-micrometer-scale (Du et al. [2002]). An alternative to the detection and destruction of individual structures is to measure the effects of the actuation on the drag resistance and adapt the system input based on this information. This brings feedback-control into play.

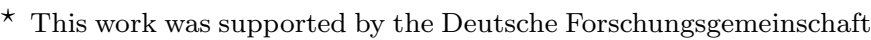
(DFG) with the grant AB65/12-1 in the Research Unit "Active Drag Reduction".
}

Actuation is then used for global jamming of the nearwall dynamics. Pressure-gradients induced in spanwise direction have proven to be successful for that task. Akhavan et al. [1993] have achieved a stabilization of this effect in simulation by the use of an oscillating wall motion. Zhao et al. [2004] and Klumpp et al. [2010] investigated spanwise travelling waves on the surface and reported significant reduction in friction drag. Subject of these simulations were fixed operating conditions, which did not include any use of feedback information. Changing inflow conditions require the rejection of disturbances in order to keep the mean friction drag at a low level. This task will be considered in this paper.

Model-based control of flows has been performed for various applications. Common control problems are the wake flow of a cylinder (e.g. Graham et al. [1999] or Tadmor et al. [2004]), the backward facing step (e.g. Ravindran [2000]) or the suppression of Tollmien-Schlichting waves (e.g. Semeraro et al. [2011]). These approaches use mode based model reduction strategies like Proper Orthogonal Decomposition (POD) and Galerkin-projection or balanced POD. However, for a turbulent boundary layer, the number of modes needed to model the near-wall dynamics is to high to be directly used in model-based control. On the other hand, the controller model needs to represent only the dynamic behaviour of the mean friction drag towards changes in actuation. A detailed reproduction of the high-frequent turbulent wall cycles is not necessary. This paper discusses a model reduction based on a limited frequency range of the CFD-simulation data, which extracts the desired actuation influence on the mean drag.

Section 2 introduces the flow setup and the numeric solution of the flow field. In section 3 the general PODGalerkin model reduction is explained. The modifications of that procedure are described in section 4. Subsequently, section 5 gives an example of a closed-loop control and the last section draws a short conclusion. 


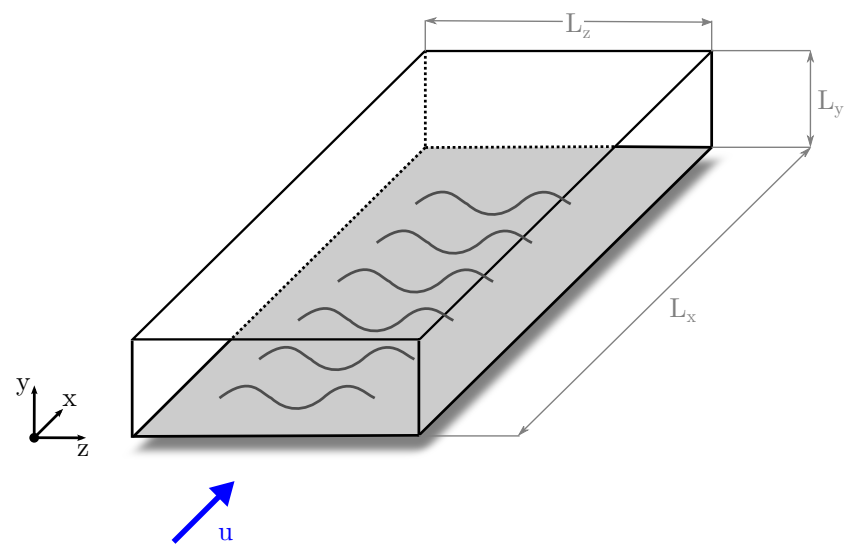

Fig. 1. Computational domain of the flow simulation

\section{FLOW SETUP}

The physical model for continuous flows are the NavierStokes equations, which result from balances of mass, momentum and energy of infinitesimal fluid elements. The free-stream Mach-number of the flow considered here is 0.2 , which allows the approximation as an incompressible flow. The resulting governing equations in non-dimensional form are the mass balance

$$
\nabla \cdot \boldsymbol{v}=0
$$

and the momentum balance

$$
\partial_{t} \boldsymbol{v}+(\boldsymbol{v} \cdot \nabla) \boldsymbol{v}=-\nabla p+\frac{1}{R e} \Delta \boldsymbol{v}+\boldsymbol{f} .
$$

Here $\boldsymbol{v}$ denotes the vector of the three velocity components $u, v$ and $w$ in direction of the Cartesian coordinates $x, y$ and $z$ respectively. The static pressure is denoted by the variable $p$ and $\boldsymbol{f}$ represents a volume force which is used for actuation. All variables are given in non-dimensional form by referring to the boundary layer thickness $\delta$ as reference length and the inflow velocity $u_{\infty}$ as reference velocity. The Reynolds-number $R e$ is the product of these reference variables divided by the kinematic viscosity of air.

An initial full-scale numerical simulation of the system is required to obtain an ensemble of snapshots, from which a reduced order model can be derived. Actuation of the system is included by the volume force $f$, which acts in form of a spanwise travelling wave. The current wave position for each spanwise coordinate $z$ is given by the function

$$
y_{W}(z, t)=\hat{A} \cdot\left(1+\sin \left(2 \pi\left(\frac{z}{\lambda(t)}-\frac{t}{T}\right)\right)\right),
$$

with the amplitude $\hat{A}=0.13$ and period time $T=$ 0.44 . The wavelength $\lambda(t)$ is varied during simulation and represents the dynamic excitation of the system. Figure 1 shows a sketch of the computational domain, which was used for the simulation. The lengths of the edges are $L_{x} / \delta=14.3, L_{y} / \delta=2.5$ and $L_{z} / \delta=2.5$. The domain is discretised with $241 \times 34 \times 76$ grid points in the $x, y$ and $z$ direction. The Reynolds-number considered here is $R e_{\delta}=10^{4}$. The data ensemble from the initial simulation consists of 2901 snapshots at a constant sampling interval of 0.01 .

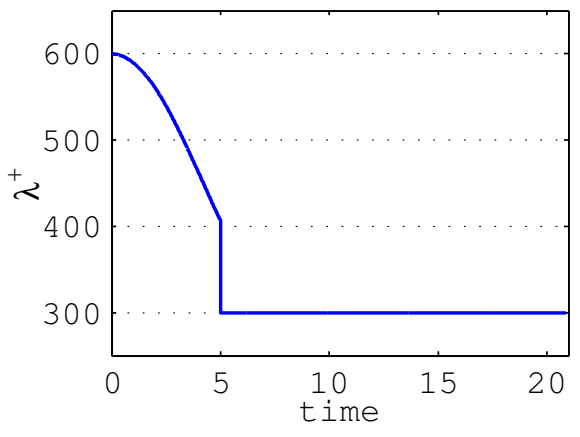

Fig. 2. Wavelength of the volume force excitation over time.

The inflow velocity distribution was generated by a synthetic turbulence generation method from Roidl et al. [2013]) and a characteristic outflow condition was used at the outlet. On the wall a no-slip boundary condition was applied and the velocity at the top of the domain was set to the free-stream velocity of $M a_{\infty}=0.2$. The solver is the same as used by Klumpp et al. [2010], which solves the compressible Navier-Stokes-Equations. Since the grid resolution is too coarse to resolve all turbulent scales directly, an implicit Large-Eddy-Simulation approach is used, where the dissipation is adjusted to take the nonresolved scales into account.

As mentioned above, the system is dynamically excited by changing the wavelength of the volume force function (2). This wavelength is depicted in figure 2 in a wall-unit scale as function of the simulation time. The conversion factor between the wall unit scale and the boundary layer scale is $R e_{\tau}=470$. The simulation is done for a fixed control volume, and the travelling wave moves from left to right through this domain. Therefore, the change of wavelength is superimposed by the movement of the wave inside the domain. To isolate the system response towards the change in wavelength, the flow is considered in a moving frame of reference for the model reduction procedure. This frame moves with the current propagation velocity of the wave, and the ensemble data is rearranged appropriately before the POD is performed.

\section{POD-GALERKIN METHOD}

To enable the use of common control design methods, the system of partial differential equations has to be reduced to a system of ordinary differential equations. This is done in two steps. At first, through the choice of an optimal set of few spatial basis functions, which approximate the known solution of the system, i.e. the ensemble data. The second step is a semi-discretisation of the governing equations based on these spatial functions. This is the standard POD-Galerkin method which is introduced shortly in the next two paragraphs.

Prior to this, let the inner product of two square-integrable functions $\mathbf{f}$ and $\mathbf{g}$ be defined as

$$
(\mathbf{f}, \mathbf{g})_{\Omega}=\sum_{i=1}^{d} \int_{\Omega} f_{i}(\mathbf{x}) g_{i}(\mathbf{x}) d \mathbf{x}
$$

where $\Omega$ describes the computational domain. 


\subsection{Proper Orthogonal Decomposition}

Using the Reynolds Decomposition, the instantaneous flow field $\boldsymbol{v}_{a}$ can be written as

$$
\boldsymbol{v}_{a}\left(\boldsymbol{x}, t_{m}\right)=\overline{\boldsymbol{v}}(\boldsymbol{x})+\boldsymbol{v}^{\prime}\left(\boldsymbol{x}, t_{m}\right),
$$

where $\overline{\boldsymbol{v}}$ represents the ensemble average of the snapshots, and $\boldsymbol{v}^{\prime}$ is the deviation from that average for each of the $M$ snapshots in the ensemble data. The POD gives a loworder approximation of these snapshots in decomposed form

$$
\boldsymbol{v}_{a}\left(\boldsymbol{x}, t_{m}\right) \approx \overline{\boldsymbol{v}}(\boldsymbol{x})+\sum_{i=1}^{N} a^{(i)}\left(t_{m}\right) \boldsymbol{\phi}^{(i)}(\boldsymbol{x})=\sum_{i=1}^{N+1} a^{(i)} \boldsymbol{\phi}^{(i)}
$$

Here $\phi(\boldsymbol{x})$ represents a spatial basis function, which is optimal for the representation of the fluctuation data $\boldsymbol{v}^{\prime}$ and $a\left(t_{m}\right)$ denotes the corresponding temporal coefficient. To simplify notation, the function arguments $\boldsymbol{x}$ and $t$ are dropped when their meaning becomes clear from the context. Furthermore the average flow field will be introduced as mode $\phi^{(N+1)}$, with a temporal coefficient identical to one for all times $a^{(N+1)} \equiv 1$. When the number of modes $N$ is increased, the approximation becomes more accurate. If $N$ equalled the number of snapshots $M$ the representation of the ensemble data would become exact, but no reduction would be achieved.

A detailed introduction of the Proper Orthogonal Decomposition requires the formal introduction of a cost function and the subsequent derivation of the eigenvalue problem for the determination of the modes. Good introductions are given by Chatterjee [2000] and Cordier and Bergmann [2008]. The calculation of the modes is accomplished here with the Method of Snapshots, which was introduced by Sirovich [1987]. With this method the temporal coefficients $a^{(i)}$ of the decomposition (5) are determined first, and the spatial basis functions are calculated from these afterwards. The temporal coefficients can be obtained as a solution of the eigenvalue problem

$$
\mathbf{C a}{ }^{(i)}=\lambda_{i} \mathbf{a}^{(i)} .
$$

Here $\lambda_{i}$ denotes the $i$-th eigenvalue and the corresponding eigenvector $\mathbf{a}^{(i)}$ contains the values of the modal coefficients for each time-step. $\mathbf{C}$ is a $M \times M$ temporal correlation matrix, whose elements are given by the inner product of two snapshots

$$
C_{k m}=\frac{1}{M}\left(\hat{\mathbf{y}}\left(\mathbf{x}, t_{k}\right), \hat{\mathbf{y}}\left(\mathbf{x}, t_{m}\right)\right)_{\Omega}
$$

With the temporal coefficients from the solution of (6), the $i$-th POD-mode is determined via

$$
\phi^{(i)}(\mathbf{x})=\frac{1}{\sqrt{M \lambda^{(i)}}} \sum_{m=1}^{M} a^{(i)}\left(t_{m}\right) \mathbf{v}^{\prime}\left(\mathbf{x}, t_{m}\right) .
$$

These modes form a set of basis functions which are mutually orthogonal, that is

$$
\left(\phi^{(i)}, \phi^{(j)}\right)_{\Omega}= \begin{cases}1 & \text { for } i=j \\ 0 & \text { for } i \neq j .\end{cases}
$$

The modes are ordered with regard to their information content towards the reproduction of the ensemble data. For any given truncation $N<M$, the reconstruction of the flow field with the first $N$ modes is the optimal approximation in the least-squares sense.
Equation (7) states that the modes are linear combinations of the snapshots. This ensures that certain features of the ensemble data are inherited by the POD modes. This applies especially for the divergence-free condition (1a) and homogeneous boundary conditions of the original solution. Therefore the approximation (5) describes a divergencefree velocity field and only the momentum balance (1b) needs to be considered as conditional equation for the reduced order model.

\subsection{Galerkin model}

The Galerkin projection is a technique for the discretisation of functional equations, such as partial or ordinary differential equations. Here, this discretisation is a necessary step to transform the PDE into an ODE system. Rewriting the momentum balance in operator notation, (1b) can be formulated as

$$
\mathcal{L}(\boldsymbol{v}, \boldsymbol{f})=\mathbf{0}
$$

with

$$
\mathcal{L}(\boldsymbol{v}, \boldsymbol{f}):=\partial_{t} \boldsymbol{v}+(\boldsymbol{v} \cdot \nabla) \boldsymbol{v}+\nabla p-\frac{1}{R e} \Delta \boldsymbol{v}-\boldsymbol{f} .
$$

Here $\boldsymbol{v}$ is the desired solution of the flow field and $\boldsymbol{f}$ the volume force. The operator $\mathcal{L}$ denotes the residual of the momentum balance. By applying a trial solution of the type $(5)$ to $\mathcal{L}$ and projecting the residual onto the set of POD basis functions, a system of ordinary differential equations is obtained. These ODEs describe the dynamics of the temporal coefficients $a^{(i)}$. Taking the orthogonality of the modes into account, the reduced-order model for the momentum balance becomes

$$
\begin{gathered}
\dot{a}^{(i)}=-\left(\boldsymbol{a}^{\mathrm{T}} 1\right) \boldsymbol{Q}_{i}\left(\begin{array}{c}
\boldsymbol{a} \\
1
\end{array}\right)+\frac{1}{R e_{\delta}} \boldsymbol{l}_{i}^{\mathrm{T}}\left(\begin{array}{c}
\boldsymbol{a} \\
1
\end{array}\right)+\left(\boldsymbol{f}, \boldsymbol{\phi}^{(i)}\right) \\
\quad \text { for } i=1, \ldots, N \\
\text { with } q_{j, k}^{(i)}=\left(\left(\boldsymbol{\phi}^{(j)} \cdot \nabla\right) \boldsymbol{\phi}^{(k)}, \boldsymbol{\phi}^{(i)}\right) \\
\text { and } l_{j}^{(i)}=\left(\Delta \boldsymbol{\phi}^{(j)}, \boldsymbol{\phi}^{(i)}\right) \\
\text { for } j, k=1, \ldots, N+1 .
\end{gathered}
$$

The non-linear structure of this ODE-System reflects the structure of the original governing equation and the number of states $N$ is determined by the number of modes used for the trial solution.

\section{FREQUENCY SELECTIVE MODEL REDUCTION}

An accurate approximation of the turbulent boundary layer dynamics requires a large number of modes due to the small turbulent scales. The blue line in figure 3 shows the relative information content from a POD of the snapshot data. More than 150 modes are necessary to reconstruct about $80 \%$ of the turbulent kinetic energy of the original ensemble data. A model of that size is too complex for real-time applications. The key for a successful model reduction is to include only those dynamics in the reduced order model that are relevant for the control task. The standard POD-Galerkin model contains dynamics of turbulent fluctuations, which are not relevant for control of the mean friction drag. To carve out the dynamics of the mean friction change in response to the variation in actuation, four steps are necessary, which are exemplified for the flow setup described in section 2 . 


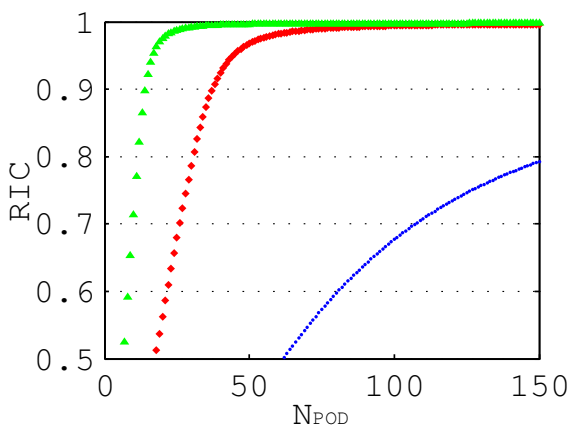

Fig. 3. Relative Information Content from POD of the original ensemble data (blue), the filtered data (red) and from the dynamic relevant subset (green).

Step 1: Determination of the relevant frequency range from analysis of the controlled variable.

Step 2: Filtering of the ensemble data with the identified relevant frequency range.

Step 3: Calculation of the POD modes from the dynamic relevant subset of the filtered ensemble data.

Step 4: Estimation of the coefficients of the resulting Galerkin model.

The first task is to examine and limit the frequency range of the control variable, such that the significant variations from the mean value are captured, whereas high frequency oscillations are suppressed. The cut-off-frequency is a design parameter for the reduced model. In this case, the frequency range of the wall-shear stress $\tau_{w}$ is examined. The blue line in figure 4 displays the variation of the wallshear stress, in the original ensemble data. Figure 5 shows the corresponding amplitude spectrum. The red lines in both figures show the same signal with a frequency range limited to 4 , which still exhibits the striking increase of friction drag during the first 5 time units.

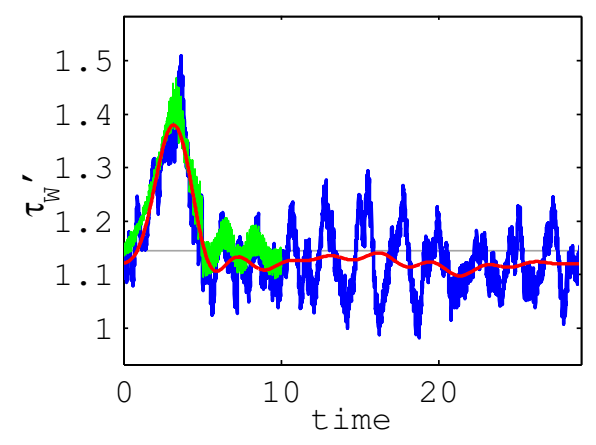

Fig. 4. Wall shear stress variation over time for the ensemble data (blue), the filtered signal (red) and the reconstruction from POD (green).

The second step requires filtering of the original ensemble data with the cut-off-frequency determined from the previous step. Here, a Butterworth low-pass filter of order 4 with a cut-off-frequency of 4 is applied to the velocity snapshots. To avoid phase delays the filter is implemented using forward-backward filtering.

In the next step, the filtered ensemble data is decomposed by POD. The red line in figure 3 shows the relative information content of this decomposition for the flow

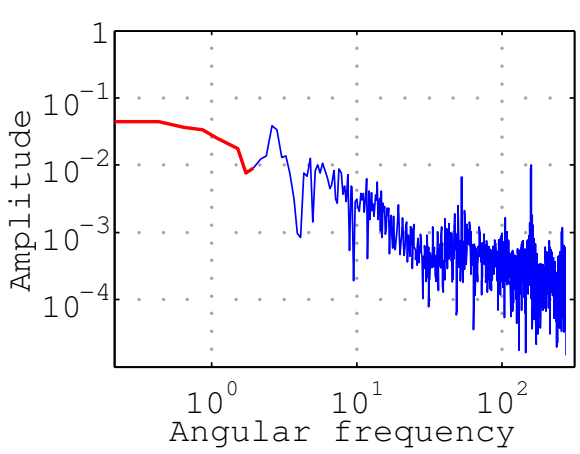

Fig. 5. Amplitude spectrum of the wall shear stress signal (blue) and the filtered frequency range (red).

case. Comparison with the blue line from the original data ensemble shows that the filtered ensemble data can be approximated with fewer modes. A restriction of the data ensemble to the first 10 time units further decreases the number of modes needed to represent the chosen dynamics. With 15 modes, the relative information content of the reconstruction is above $90 \%$. This number of modes will be used for the reduced order model. The wall-shear stress signal from the velocity field, which is reconstructed with this POD-approximation, is given by the green line in figure 4 .

The last step for the reduced-order modelling is the identification of the model parameter values. In the standard POD-Galerkin approach these are determined by the Galerkin-projection. Due to the nonlinearity of the governing equations, the neglected turbulent scales influence the mean field behaviour. If the decomposition is performed on the non-filtered snapshot data, this influence would be incorporated in the reduced order model. However, for the POD modes from the filtered ensemble, the Galerkin projection generates parameter values, which do not represent the influence of the neglected scales. Therefore, the model parameter values are identified using the modal coefficients from the POD. This approach was introduced by Couplet et al. [2005] and later adapted by Cordier et al. [2010]. For ease of notation, the right-hand-side of the reduced order model (10) is introduced in operator notation

$$
\mathcal{N}_{i}(\boldsymbol{p}, \boldsymbol{a})=-\left(\boldsymbol{a}^{\mathrm{T}} 1\right) \boldsymbol{Q}_{i}\left(\begin{array}{c}
\boldsymbol{a} \\
1
\end{array}\right)+\frac{1}{R e_{\delta}} \boldsymbol{l}_{i}^{\mathrm{T}}\left(\begin{array}{c}
\boldsymbol{a} \\
1
\end{array}\right)+p_{0}^{(i)} \cdot f(t) .
$$

The vector $\boldsymbol{p}$ denotes the parameter values of the matrix $\boldsymbol{Q}_{i}$ and the vector $\boldsymbol{l}_{i}$. Furthermore it is assumed that the actuation function can be split up into a time dependent part $f(t)$ and a spatial distribution factor. The inner product of the latter one with the $i$-th mode is considered as the actuation parameter $p_{0}^{(i)}$.

Since the modal coefficients are known from the POD, the estimation of the model parameters can be reduced to the optimization problem

$$
\begin{aligned}
& \min _{\boldsymbol{p}} \mathcal{J}_{\gamma}(\boldsymbol{p}) \\
& \quad \text { with } \quad \mathcal{J}_{\gamma}(\boldsymbol{p})=\left\|\dot{a}^{(i)}-\mathcal{N}_{i}(\boldsymbol{p}, \boldsymbol{a})\right\|_{2}+\gamma\left\|\boldsymbol{p}-\boldsymbol{p}_{G}\right\|_{2}
\end{aligned}
$$

which can be solved independently for each of the $N$ model equations. The time derivatives of the modal coefficients $\dot{a}^{(i)}$ are computed via numerical differentiation. The 


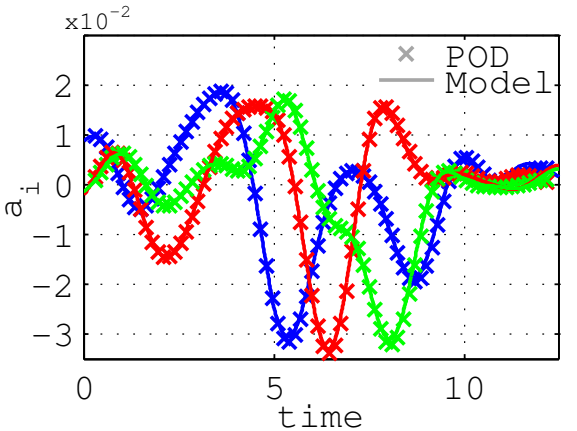

Fig. 6. Comparison of the first three states of the identified Galerkin model with the corresponding modal coefficients of the POD.

second term in the cost function represents a Thikonovregularisation to improve the numeric condition of the problem. $\boldsymbol{p}_{G}$ represents the parameter vector obtained from the Galerkin-projection. The weight factor $\gamma$ for this penalty term is chosen as $10^{-5}$.

Note that the cost function is quadratic with regard to the model states, but depends only linear on the parameter values. The solution of the optimization problem (12) can be determined by the computation of $N$ linear equation systems of the form

$$
\left(\boldsymbol{A}^{\mathrm{T}} \boldsymbol{A}+\gamma \boldsymbol{I}\right) \boldsymbol{p}=\left(\boldsymbol{A}^{\mathrm{T}} \boldsymbol{b}+\gamma \boldsymbol{p}_{G}\right) .
$$

The structure of the matrix $\boldsymbol{A}$ and the vector $\boldsymbol{b}$ is given by Cordier et al. [2010]. In spite of the regularization, a simultaneous determination of the parameters belonging to the quadratic convection term and the linear diffusion term is still numerically ill-conditioned. Therefore the matching of the parameters is done consecutively. First the parameters of the diffusion term and the actuation influence are determined. Second, the parameters of the convection term are adapted.

For the parameter identification the actuation function in (11) should match the actuation of the initial system simulation. However, in this case the wall-shear-stress, as depicted in figure 4 , seems to depend on the change of the wavelength rather than on the absolute value of the wavelength itself. For that reason the rate of change of the wave function was used for the parameter identification. Thus the input signal is

$$
f(t)=-\hat{A} \cdot \frac{2 \pi \lambda_{0}}{(\lambda(t))^{2}} \cdot \dot{\lambda}(t) \cdot \cos \left(\frac{2 \pi \lambda_{0}}{\lambda(t)}\right),
$$

where $\lambda_{0}$ is the width of the moving reference frame in $z$ direction. Figure 6 shows the model states of the identified Galerkin model in comparison to the modal coefficients of the POD. Both data agree very well, which indicates that the model structure suits the set of training data. Identification approaches with linear models were not able to fit the training data that well.

\section{RESULTS}

\subsection{Validation}

The model is validated by comparing the model prediction to those parts of the ensemble data, that were not used for the parameter estimation. The red curve in figure 7 shows

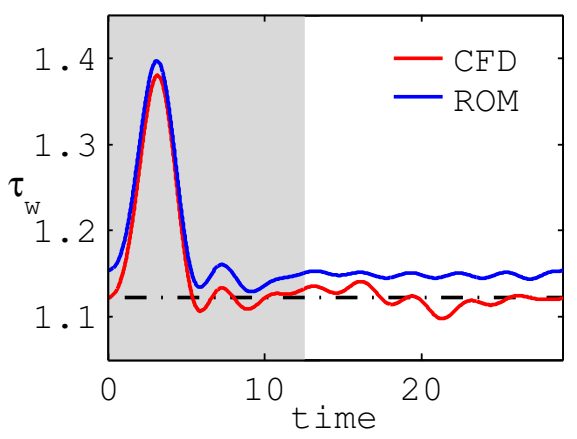

Fig. 7. Wall-shear stress signal from the numerical solution (red) and from the reduced-order model (blue). The shaded background indicates the data, that was used for parameter identification.

the wall shear-stress from the initial numerical solution. The blue line displays the wall-shear stress as predicted with the reduced-order-model. The grey background indicates those parts that were used for the training of the reduced-order model. To eliminate noise effects which result from the discretisation of the flow field, the displayed shear-stress signals are limited to the frequency range of 4. The mean friction drag is slightly overestimated by the reduced-order model but the dynamics match the behaviour of the ensemble data.

\subsection{Closed Loop Control}

A closed-loop simulation with model predictive control is demonstrated as an application example. The plant model in this simulation is represented by an reduced order model with 25 states, which was derived by the procedure outlined above. For the controller model 15 states where used. To include persisting changes in friction drag, both models were equipped with an additional integrating state that influences the mean field. The measurement information for the controller model is the wall-shear stress signal at the current time step.

The controller consists of an Extended Kalman Filter (EKF) and a linear model predictive controller (MPC), as formulated by Maciejowski [2002]. Both model and filter use the 15 state reduced-order model. The prediction in the EKF is done with the non-linear model, whereas for the correction step and for the MPC a local linearisation of the model is used. This linearisation is performed analytically from (10) at each sampling time. The resulting optimisation problem for the MPC is solved with the qp-Solver qpOases from Ferreau et al. [2008].

The simulation result is depicted in figure 8. The controller quickly responds to the unknown output disturbance and is able to recover the original level of friction drag. The limitations in the plant input are taken into account by the optimization. The slow convergence of the shear-stress towards the mean level after the disturbance results from a difference in the static gain of the plant and controller model.

\section{CONCLUSION}

The control application that was shown here represents a single SISO problem. A direct identification of the 


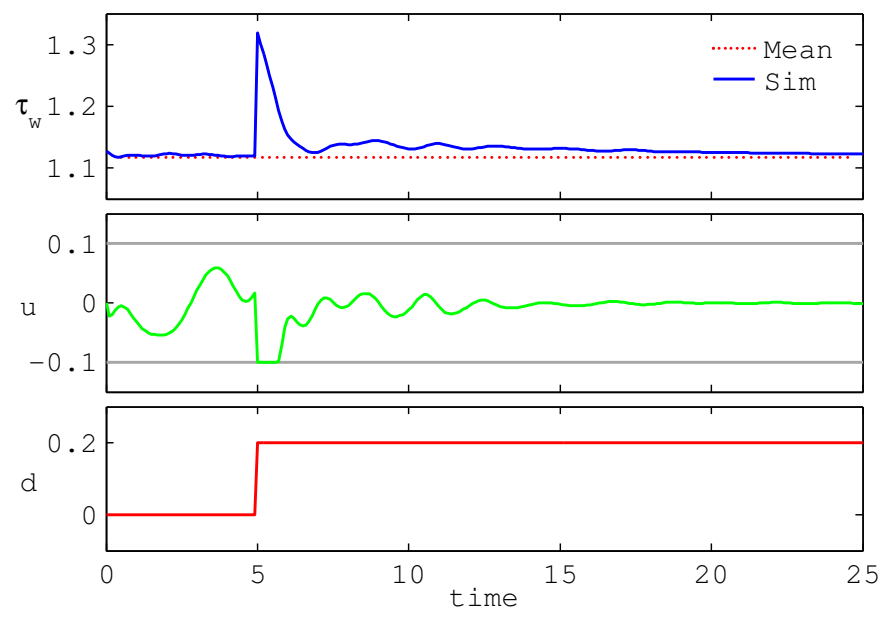

Fig. 8. Closed-loop simulation result. Depicted are the controlled wall shear-stress (blue) and the reference trajectory (red dots), the plant input $u$ (green) with limitations (grey) and the output disturbance $d$ (red).

transfer function between the variation in wavelength and the resulting change in friction drag would be an obvious choice to obtain a plant model. The POD-Galerkin procedure seems to be disproportionate for such a control problem. However, this method shows two advantages in comparison to an I/O-model. First, the non-linear structure of the reduced-model is specified by the physical governing equations. Indeed, the quadratic model fitted the training data better than a linear model of comparable size. The second and more important advantage is the information that is contained in the reduced-order model. The Galerkin model is able to reconstruct the whole flow field that was used to generate the POD basis. This becomes important for real-world applications in which the wall-shear stress usually cannot be measured directly. In that case, the Galerkin-model offers the possibility to observe the friction drag e.g. from velocity measurements. In that context, the presented frequency limitation is a proper method to constrain the reduced-order model, such that only the desired dynamics are incorporated.

The next steps are a further validation of the reduced order model with different excitation signals and a co-simulation of the controller with the numerical flow solver.

\section{REFERENCES}

R. Akhavan, W.J. Jung, and N. Mangiavacchi. Turbulence control in wall-bounded flows by spanwise oscillations. Applied Scientific Research, 51:299-303, 1993.

A. Chatterjee. An introduction to the proper orthogonal decomposition. Current Science, 78:808-817, 2000.

H. Choi, P. Moin, and J. Kim. Active turbulence control for drag reduction in wall-bounded flows. J. Fluid Mech., 262:75-110, 1994.

L. Cordier and M. Bergmann. Proper orthogonal decomposition: an overview. In Post-Processing of Numerical and Experimental Data. von Karman Institute for Fluid Dynamics, 2008.

L. Cordier, B. Abou El Majd, and J. Favier. Calibration of POD reduced-order models using tikhonov regularization. International Journal for Numerical Methods in Fluids, 63:269-296, 2010.
M. Couplet, C. Basedevant, and P. Sagaut. Calibrated reduced-order POD-Galerkin system for fluid flow modelling. Journal of Computational Physics, 207:192-220, 2005.

Y. Du, V. Symeonidis, and G. E. Karniadakis. Drag reduction in wall-bounded turbulence via a transverse travelling wave. J. Fluid Mech., 457:1-34, 2002.

H.J. Ferreau, H.G. Bock, and M. Diehl. An online active set strategy to overcome the limitations of explicit MPC. International Journal of Robust and Nonlinear Control, 18(8):816-830, 2008.

B. Frohnapfel, Y. Hasegawa, and M. Quadrio. Money versus time: evaluation of flow control in terms of energy consumption and convenience. J. Fluid Mech., 700:406418, 2012.

W.R. Graham, J. Peraire, and K.Y. Tang. Optimal control of vortex shedding using low-order models. Part 1 - Open loop model development. International Journal for Numerical Methods in Engineering, 44:945-972, 1999.

Ph. Holmes, J. L. Lumley, and G. Berkooz. Turbulence, Coherent Structures, Dynamical Systems and Symmetry. Cambridge University Press, 1996.

S. Klumpp, M. Meinke, and W. Schröder. Drag reduction by spanwise transversal surface waves. Journal of Turbulence, 11(22):1-13, 2010.

J.M. Maciejowski. Predictive control with Constraints. Prentice Hall, Harlow, UK, 2002.

R. Rathnasingham and K. S. Breuer. Active control of turbulent boundary layers. J. Fluid Mech., 495:209-233, 2003.

S.S. Ravindran. A reduced-order approach for optimal control of fluids using proper orthogonal decomposition. International Journal for Numerical Methods in Fluids, 34:425-448, 2000.

B. Roidl, M. Meinke, and W. Schröder. A reformulated synthetic turbulence generation method for a zonal RANS-LES method and its application to zero-pressure gradient boundary layers. International Journal of Heat and Fluid Flow, 44:28-40, 2013.

O. Semeraro, Sh. Bagheri, L Brandt, and D. S. Henningson. Feedback control of three-dimensional optimal disturbances using reduced-order-models. J. Fluid Mech., 677:63-102, 2011.

L. Sirovich. Turbulence and the dynamics of coherent structures Part I: Coherent structures. Quarterly of Applied Mathematics, XLV(3):561-571, 1987.

G. Tadmor, B. R. Noack, M. Morzyński, and S. Siegel. Low-dimensional models for feedback flow control. Part II: Controller design and dynamic estimation. In 2nd AIAA Flow Control Conference, Portland, Oregon, USA, 2004.

M. J. Walsh. Turbulent boundary layer drag reduction using riblets. In 20th AIAA Aerospace Sciences Meeting, Orlando, Florida, USA, 1982.

T. Yoshino, Y. Suzuki, and N. Kasagi. Drag reduction of turbulence air channel flow with distributed micro sensors and actuators. Journal of Fluid Science and Technology, 3(1):137-148, 2008.

H. Zhao, J.-Z. Wu, and J.-S. Luo. Turbulent drag reduction by traveling wave of flexible wall. Fluid Dynamics Research, 34:175-198, 2004. 